\title{
Design and characterization of in-plane MEMS yaw rate sensor
}

\author{
K P VENKATESH, NISHAD PATIL, ASHOK KUMAR PANDEY \\ and RUDRA PRATAP*
}

CranesSci MEMS Laboratory, Department of Mechanical Engineering, Indian Institute of Science, Bangalore 560012

e-mail: pratap@mecheng.iisc.ernet.in

\begin{abstract}
In this paper, we present the design and characterization of a vibratory yaw rate MEMS sensor that uses in-plane motion for both actuation and sensing. The design criterion for the rate sensor is based on a high sensitivity and low bandwidth. The required sensitivity of the yaw rate sensor is attained by using the inplane motion in which the dominant damping mechanism is the fluid loss due to slide film damping i.e. two-three orders of magnitude less than the squeeze-film damping in other rate sensors with out-of-plane motion. The low bandwidth is achieved by matching the drive and the sense mode frequencies. Based on these factors, the yaw rate sensor is designed and finally realized using surface micromachining. The inplane motion of the sensor is experimentally characterized to determine the sense and the drive mode frequencies, and corresponding damping ratios. It is found that the experimental results match well with the numerical and the analytical models with less than $5 \%$ error in frequencies measurements. The measured quality factor of the sensor is approximately 467, which is two orders of magnitude higher than that for a similar rate sensor with out-of-plane sense direction.
\end{abstract}

Keywords. Rate sensor; drive mode; sense mode; sensitivity; bandwidth; PMA.

\section{Introduction}

Micromachined inertial sensors consisting of accelerometers and gyroscopes are one of the most important types of silicon-based sensors due to much broader spectrum of their applications (Yazdi et al 1998). Micromachined gyroscopes measuring the angular rate have attracted a lot of attention during the past few years for several applications in which they can be integrated with the other miniaturized sensors such as MEMS accelerometers (Bao 2000). One such application in which the rate sensors are used with micromachined accelerometers is to provide heading information for inertial navigation purposes and for some consumer electronic applications such as video camera stabilization, virtual reality and robotic applications (Yazdi et al 1998; Maluf 2000; Patil 2006).

In this paper, we discuss the design and characterization of a vibratory yaw rate MEMS sensor that uses in-plane motion for both actuation and sensing. Like most of the vibratory

*For correspondence 
angular rate sensors, this sensor is also based on the principle of Coriolis acceleration induced oscillations in the sense direction (Bao 2000). In most angular rate sensors, the drive direction is in-plane and the sense direction is out-of-plane. One of the major drawbacks of such design is the large damping due to squeeze-film flow which reduces the sensitivity of the device (Kwok 1999). This particular drawback can be mended to a large extent by taking the sense direction to be in-plane. It is because the fluid damping for in-plane motion is mainly due to the slide-film damping that is two to three orders of magnitude smaller than the squeeze-film damping (Kwok 1999; Jeong et al 2004) in the out-of-plane motion. It also turns out that the in-plane design is least sensitive to residual stresses that are a major cause of frequency mismatch between the modes of interest and thus the bandwidth of the sensor. The sensor discussed here is designed for a constant angular rate signal. Hence, the design criterion is based on a low bandwidth and high sensitivity. The yaw rate sensor thus designed is realized using surface micromachining. The realized structure is characterized by using PMA (Planar Motion Analyzer) - a Polytec product to characterize the in-plane motion (Polytec, Andojo \& Tay 2005). From the experimental results, we find the sense and the drive mode frequencies, and the damping ratio using the half power point method. Finally, we compare the experimental results with the numerical and the analytical models.

\section{Operating principle}

The operation of all types of vibratory gyroscopes is based on the energy transfer between two modes of vibration of the moving structure which are caused due to Coriolis acceleration (Yazdi et al 1998; Bao 2000; Patil 2000). The Coriolis acceleration is an apparent acceleration that arises in a rotating frame of reference and is proportional to the rate of rotation (Bao 2000).

To describe the operating principle, the vibratory gyroscope can be represented by a two degree of freedom spring-mass-damper system as shown in figure 1. In this system, the proofmass acts as a moving mass while the beams anchored to the substrate provide required stiffness to the system. When the proof mass is excited in the $x$-direction (drive direction) of the gyroscopic frame by the force $F_{d}$ and an external rotation about the $z$-direction (into the plane of the paper), the induced Coriolis force causes the mass to oscillate in the $y$-direction (i.e. in the sense direction). Generally, the magnitude of the sense oscillation is proportional to the rotational rate of the device (Bao 2000; Patil 2006).

If $x$ is the displacement in drive direction, $y$ is the displacement in sense direction and $\Omega$ is the input angular rate, the equation of motion for the system can be written as (Fedder 1994; Bao 2000; Patil 2006):

$$
\begin{aligned}
& m \ddot{x}+c_{x} \dot{x}+k_{x} x-2 m \Omega \dot{y}=F_{d} \\
& \ddot{y}+c_{y} \dot{y}+k_{y} y-2 m \Omega \dot{x}=0,
\end{aligned}
$$

where $m$ is the proof mass, $c_{x}$ and $c_{y}$ are the damping constants due to in-plane motions in $x$ and $y$-directions, respectively, and $k_{x}$ and $k_{y}$ are the corresponding stiffnesses. The terms $2 m \Omega \dot{x}$ and $2 m \Omega \dot{y}$ in eqns. (1) and (2) are the rotation-induced Coriolis force terms, which establish the dynamic coupling between the two modes of vibration (Patil 2006). When the stiffness values in the drive and the sense modes are matched, the resonant frequencies of the two modes become the same. If the system is driven at its resonant frequency using harmonic excitation $\left(F_{d}=F_{0} \sin (\omega t)\right)$ then the rotation induced Coriolis force excites the system into 


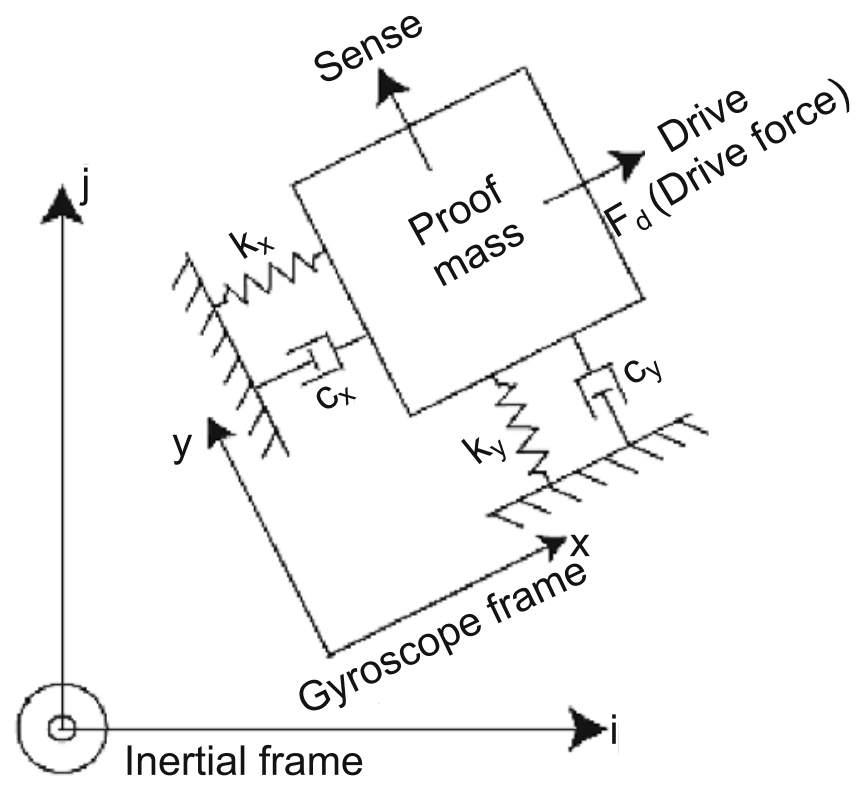

Figure 1. Operating principle of a vibratory gyroscope.

resonance in the sense direction. The resulting oscillation amplitude of the sense motion is proportional to the Coriolis force and, thus, the angular velocity can be measured.

\section{Performance characteristics}

The prime performance parameters of the gyroscope are resolution, dynamic range, bandwidth, sensitivity, scale factor, noise floor, cross axis sensitivity and drift. The bandwidth defines how the gyroscope responds to variations in angular rate. Hence bandwidth gives the maximum input frequency for which the angular rate measurements are accurate. The sensitivity is defined as the ratio of the capacitance change to the applied angular rate. Both these parameters are decided by the difference between the drive and sense mode frequencies and the quality factors in drive and sense directions. If the difference between the drive and sense mode frequencies is very small then sensitivity of the device increases at the cost of its bandwidth. In the present context we design a yaw rate sensor with low bandwidth and high sensitivity, and characterize the same to find its drive and sense mode frequencies, which dictate the performance characteristics.

\section{Structural design}

The novelty of the present design lies in the decoupling of the drive and the sense modes (Fedder 1994; Alper \& Akin 2002). The structure shown in figure 2 may be modelled as a 2DOF system having two resonance frequencies, the driving frequency $\omega_{x}=\sqrt{k_{x} / m}$ and the sense frequency $\omega_{y}=\sqrt{k_{y} / m}$. When the stiffness of the beams are made equal, the sense and drive mode frequencies are matched. If the system is driven with a force $F_{d}$ with the natural 


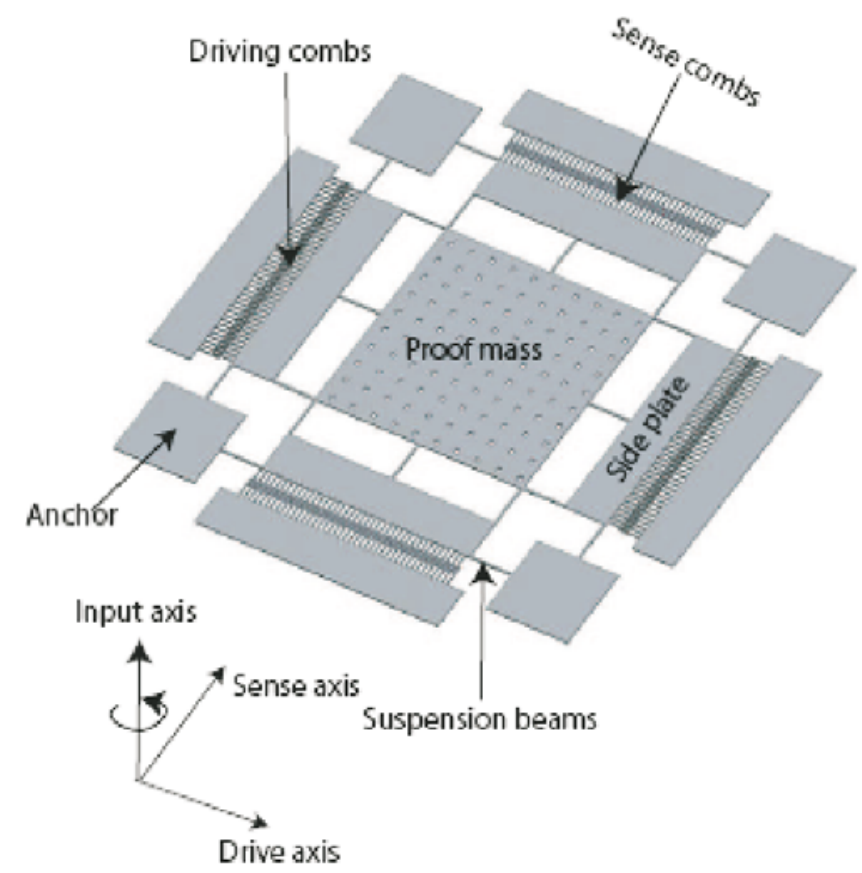

Figure 2. Structure of in-plane gyroscope.

frequency of the system then it oscillates at resonance in the drive mode, thus maximizing the oscillation amplitude in that direction. Now, if the system is subjected to a rotation about the $z$-axis, the resultant Coriolis force excites the sense mode in resonance, thus, maximizing the response amplitude. The $z$-axis gyroscope as explained above is commonly referred to as the yaw rate sensor. So, the yaw sensor has both the sense and drive modes in the plane of the device. The structural design of one such yaw rate sensor is explained below.

Figure 2 shows the structure of an in-plane yaw rate sensor. The design of this sensor has a symmetric suspension. Unlike the other designs in which the comb fingers are provided on the sides of proof mass, the comb fingers in this case are placed on separate plates as shown in figure 2. These plates are connected to the proof-mass through separate suspended beams. The purpose of using this arrangement is to uncouple the motion of side plates from the proof mass. In the drive mode, the beams along the drive direction, which connect the proof mass with the side plates, do not deform due to high axial stiffness. Similarly, in the sense mode, the displacements of the beams along the drive direction are negligible. This property of the structure leads to the decoupling of the oscillation modes as the comb-fingers on the side plates move only due to the Coriolis force, but not during drive motion. The capacitance change due to the sense motion is picked up by comb fingers provided along the sense direction.

\section{Microfabrication process}

The fabrication of the yaw rate sensor shown in figure 2 involves well-known steps of surface micromachining (Madou 1997). First, the poly layer of thickness $0.5 \mu \mathrm{m}$ is deposited using LPCVD process which acts as an electrode layer. Then the electrode layer is patterned using dry etching. The sacrificial layer is obtained by depositing PSG or oxide of thickness $1.6 \mu \mathrm{m}$ using LPCVD process. Patterning of the oxide layer is done using the mask for providing 


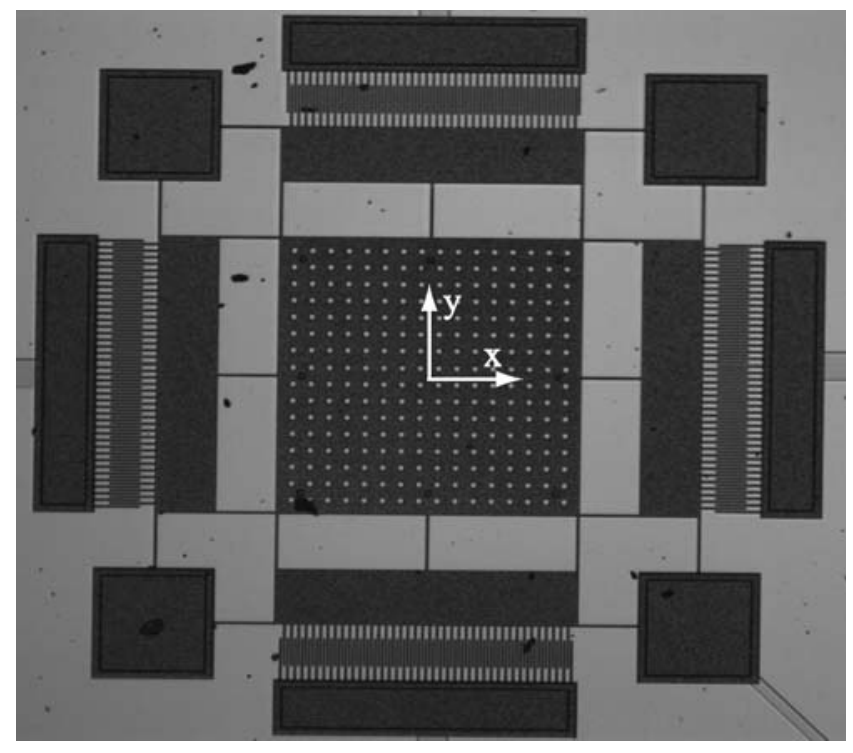

Figure 3. An SEM image of yaw rate sensor.

anchors. The structure layer is obtained by depositing polysilicon of thickness $3.5 \mu \mathrm{m}$ using LPCVD process. Then a metal layer of thickness $0.6 \mu \mathrm{m}$ is deposited on the top of the structural layer. The bond pads are provided by patterning the metal layer. Subsequently, the masking oxide layer is deposited using PECVD process. The released etch holes in the structural layer are obtained by patterning the masking layer and then the structural layer. Finally, the released structure is obtained by dissolving the sacrificial oxide layer in $49 \% \mathrm{HF}$ at room temperature.

The in-situ inspection of the released structure using SEM images of the structure shown in figure 3 reveals that the final structure is released properly. This fact can be checked by performing dynamic characterization which is discussed in the next section. It is observed that the comb fingers as well as the suspended beams are straight and thus giving a clue of negligible residual stress in the final structure. Based on this analysis, the analytical and numerical models, which are described in the subsequent sections, are carried out without considering the effect of residual stresses.

\section{Analytical computation of natural frequency}

As mentioned earlier, the computation of drive and sense mode frequencies are the deciding parameters for the sensitivity and bandwidth of the rate sensor. In this section, we analytically compute the natural frequencies in the drive and sense modes. The accurate computation of the natural frequency of any dynamic system is dependent on the accuracy with which its net spring constant and the effective mass are determined. For a given spring constant $K$ and the effective mass $M$ corresponding to any degree of freedom, the natural angular frequency may be obtained from:

$$
\omega_{n}=\sqrt{\frac{K}{M}} .
$$


Table 1. Stiffness calculations.

\begin{tabular}{ll}
\hline Parameter & Value \\
\hline Length of the beam, $\left(l_{b}\right)$ & $100 \mu \mathrm{m}$ \\
Width of the beam, $\left(w_{b}\right)$ & $5 \mu \mathrm{m}$ \\
Thickness of the beam, $\left(t_{b}\right)$ & $3.5 \mu \mathrm{m}$ \\
Young's modulus of material, $E$ & $160 \mathrm{GPa}$ \\
Stiffness in drive direction, $K_{d}$ & $700 \cdot 0 \mathrm{~N} / \mathrm{m}$ \\
Stiffness in sense direction, $K_{s}$ & $700 \cdot 0 \mathrm{~N} / \mathrm{m}$ \\
\hline
\end{tabular}

Now, if $K_{d}$ and $K_{s}$ are the stiffnesses provided by the suspended beams in the drive and the sense directions, and $M_{d}$ and $M_{s}$ are their corresponding effective masses, then their angular natural frequencies are given by $\omega_{d}=\sqrt{\frac{K_{d}}{M_{d}}}$ and $\omega_{s}=\sqrt{\frac{K_{s}}{M_{s}}}$, respectively. Since the length, width, thickness and the material of the all the beams are same and so are their effective masses in the drive as well as the sense mode, the stiffness constants are also equal. The beams involved in the sense and the drive modes are guided cantilever beams and they are ten in numbers, so the expression of net stiffness in either type of motion is given by (Fedder 1994; Patil 2006; Alper \& Akin 2002):

$$
K_{d}=K_{s}=\frac{10 E t_{b} w_{b}^{3}}{t_{b}^{3}} .
$$

The values of the net stiffness in either mode for the fabricated structure are mentioned in table 1. The value of the effective mass may be obtained from (Patil 2006; Alper \& Akin 2002):

$$
M_{\text {eff }}=M_{\text {proof mass }}+M_{\text {side plates }}+M_{\text {comb fingers }}+M_{\text {beams }}-M_{\text {etch holes }} .
$$

The net mass of the system and its constituents are tabulated in table 2 .

Now, based on the above values of the stiffness and the effective mass, the frequencies corresponding to the drive and the sense modes are computed from eqn. (3). The values of the natural frequencies are tabulated in table 3.

Table 2. Effective mass calculations.

\begin{tabular}{llc}
\hline Entity & Formula & Value $(\mu \mathrm{g})$ \\
\hline Proof mass, $M_{P}$ & $\rho l_{p} w_{p} t$ & 2.012 \\
Side plate, $M_{s p}$ & $4 \rho l_{s p} w_{s p} t$ & 0.805 \\
Comb fingers, $M_{c}$ & $N_{c} \rho l_{c} w_{c} t$ & 0.193 \\
Beams, $M_{b}$ & $N_{b} \frac{13}{35} \rho l_{b} w_{b} t$ & 0.0012 \\
Etch holes, $M_{e}$ & $N_{h} \rho W_{h} L_{h}$ & 0.261 \\
Effective mass & & 2.791 \\
\hline
\end{tabular}


Table 3. Natural frequency calculations.

\begin{tabular}{lcc}
\hline Parameter & Drive mode & Sense mode \\
\hline Stiffness & $700 \cdot 0 \mathrm{~N} / \mathrm{m}$ & $700 \cdot 0 \mathrm{~N} / \mathrm{m}$ \\
Effective mass & $2.8 \mu \mathrm{g}$ & $2.8 \mu \mathrm{g}$ \\
Frequency & $79.7 \mathrm{kHz}$ & $79.7 \mathrm{kHz}$ \\
\hline
\end{tabular}

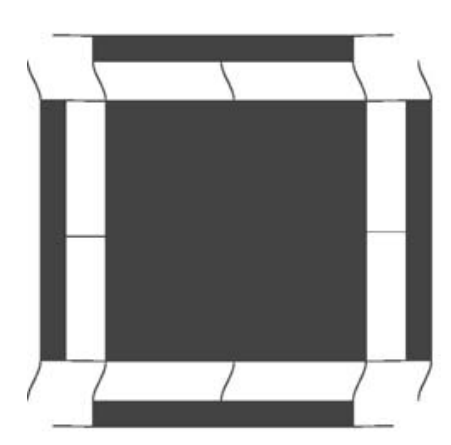

(a) Drive mode

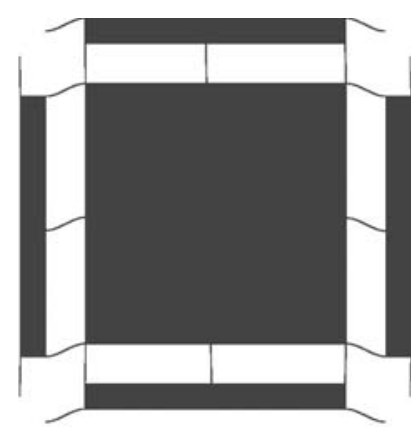

(b) Sense mode

Figure 4. The mode shape.

\section{Numerical studies}

The numerical results are obtained by simulating the test structure in finite-element software, ANSYS (2004). The numerical computation of natural frequencies and the mode shapes of the drive and the sense modes involve three main steps. In the first step, the 2-D model is created as shown in figure 2 and meshed with 25000 eight-noded quadrilateral elements. The zero-displacement and zero rotation conditions are applied on the fixed edges of the beams. Then, the solution step is performed by carrying out modal analysis using Block-Lagrange solver in ANSYS. Finally, the natural frequencies and the corresponding mode shapes (shown in figure 4) for the drive and the sense modes are extracted in the post-processing step. The numerical values of frequencies corresponding to the drive and the sense modes are mentioned in table 4.

\section{Experimental studies}

In this section, experiments are performed to characterize the yaw rate sensor using planar motion analyzer (PMA) which makes use of stroboscopic principle to measure the in-plane displacement of the device. The operating principle of PMA is schematically described in

Table 4. Numerical results.

\begin{tabular}{ccc}
\hline \multirow{2}{*}{ Frequency } & Drive mode & Sense mode \\
$79.8 \mathrm{kHz}$ & $79.8 \mathrm{kHz}$ \\
\hline
\end{tabular}



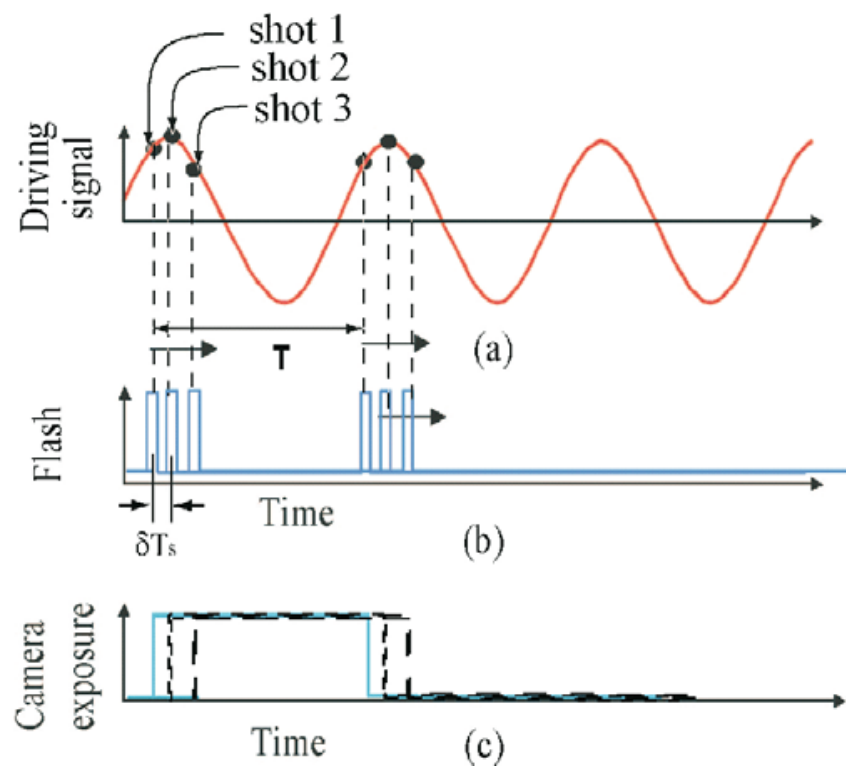

Figure 5. Working principle of test set-up.

figure 5 which is based on stroboscopic video microscopy that uses the normal CCD camera as described by Andojo \& Tay (2005). The periodic motion of the device is first captured by an illumination source such as a strobed light (flash) for certain durations. During that time, the short light pulses, i.e. shots per period, may record the position of the device at precise angles. Since a camera shot is a sequence of flashes within a camera exposure time, the motion of the device over a period can be measured by shifting the timing of those pulses as shown in figure 5. For each shot, the total number of flashes (periods) are illuminated for a specific flash duration (generally, for a ns). If $\delta T_{s}$ is the time between two shots, which is the cyclic duration of the camera-framing rate, the phase delay of the strobe illumination $\left(t_{\text {phase delay }}\right)$ with respect to the driving signal may be adjusted by setting the time delay between the shots from the relationship (Andojo \& Tay 2005):

$$
T_{\text {shot }}=n_{p} T_{\text {driving }}+t_{\text {phase delay }},
$$

where $T_{\text {shot }}$ is the total length of the sequence of flashes (in s), $n_{p}$ is the number of periods to be observed (number of flashes per shot), and $T_{\text {driving }}$ is the period of the driving signal (in $s$ ).

For a given shot time, $T_{\text {shot }}$, illumination can maximize the number of flashes per shot $\left(n_{f}\right)$ as follows (Andojo \& Tay 2005):

$$
n_{f} \alpha \frac{T_{\text {camera }}}{T_{\text {shot }}}
$$

Therefore, to obtain frequency measurement with PMA, the flash frequency should be selected properly to accurately capture the image of the moving device which oscillates with the driving frequency.

Based on the above description, the measurement procedure involves the following settings in the PMA software: the mode that has to be measured, the excitation and the flash frequency, the time of illumination and the excitation voltage. The subsequent steps involve image recording, processing and the displacement measurement by matching the patterns obtained 


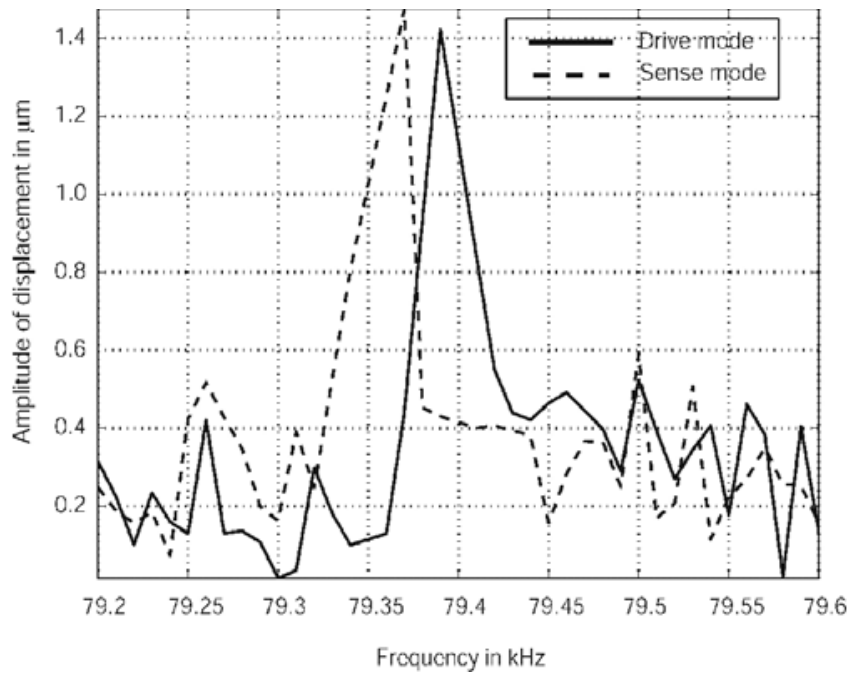

Figure 6. Frequency response curve for the drive and sense motion obtained using PMA.

during the measurements. Finally, the results are obtained in the time as well as the frequency domain.

For the device under testing, a sinusoidal signal of the driving frequency is applied across the driving electrodes to get the vibratory motion of the structure with same frequency. Here, the input voltage for exciting the structure is taken as $V_{\text {input }}=8 \pm 2 \mathrm{~V}$. Then, the frequency is swept over a range around the resonance. Since, the theoretically computed resonance frequency is found to be around $79 \mathrm{kHz}$, the frequency range is taken from 79 to $80 \mathrm{kHz}$. Figure 6 shows the frequency response curves of yaw rate sensor in the drive and the sense modes. The experimental values of the resonance frequencies corresponding to the drive and the sense modes are $79.39 \mathrm{kHz}$ and $79.37 \mathrm{kHz}$, respectively. A difference of $20 \mathrm{~Hz}$ observed between the drive and the sense modes frequencies gives additional advantage of having small bandwidth, thus improving the sensor performance. This difference can be negligible if it is compared to the drive and the sense frequencies. The source of this difference is because of the difference in the dimensions of suspended beams due to fabrication effect. Figure 7 shows

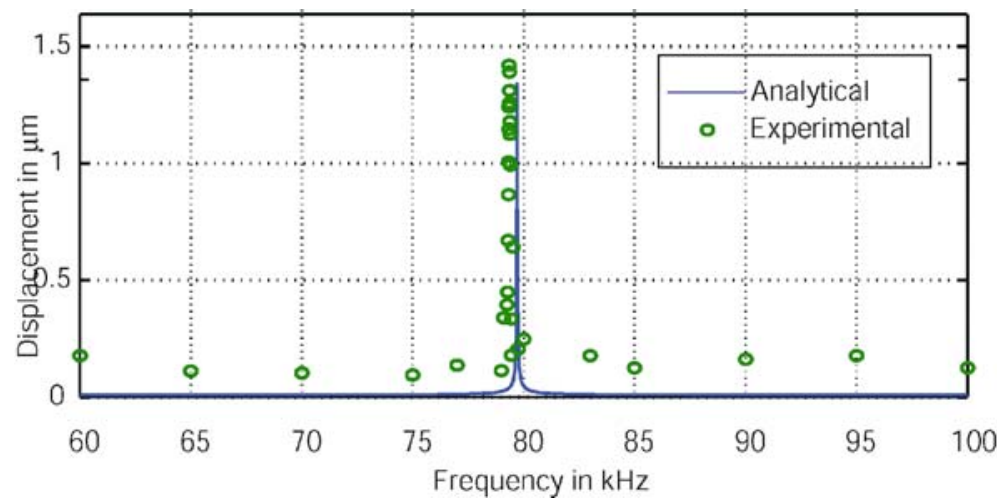

Figure 7. Comparison between analytical and experimental results. 
the comparison between analytical and experimental results. It is found that the experimental results match with the analytical result very well. Finally, the quality factor corresponding to the drive and the sense motions are measured using half-power point method, which are found to be approximately the same and equal to $466 \cdot 76$.

\section{Conclusions}

A yaw rate sensor has been designed and fabricated using a $3.5 \mu \mathrm{m}$ surface micromachining process. A symmetrical design has been employed to ensure that residual stresses, if any due to fabrication process affect both the drive and sense modes equally, allowing for a high sensitivity. However, SEM studies show that the flexures, proof-mass, side plates and combfingers are stress free. The sensor has been characterized by using a planar motion analyzer. The experimental studies show that there is a $20 \mathrm{~Hz}$ difference between the drive and the sense mode frequencies which are mainly due to small differences between the widths of the beams in the sense and drive directions.

The quality factor has been measured to be 466.76 which is two orders of magnitude higher than that for out-of-plane sensing, because the loss is mainly due to Couette flow damping, and there is no squeeze film flow which is the major source of loss in the majority of MEMS devices. The bandwidth of this sensor which is a function of the difference in the drive and sense mode frequencies is small, as the frequency mismatch is $20 \mathrm{~Hz}$. This frequency mismatch can be further tuned to achieve adequate bandwidth with the help of electromechanical feedback implemented with the aid of sophisticated electronics.

This work was funded by the Department of Science and Technology, New Delhi, India and National Program of Smart Materials, Bangalore.

\section{References}

Alper S, Akin T 2002 A symmetric surface Micromachined gyroscope with decoupled oscillation modes. Sensors and Actuators A97-98: 347-358

Andojo Ongkodjojo, Francis E H Tay 2005 Characterization of micromached devices using planar motion analyzer (PMA). Proc. IEEE SENSORS'2005 30: 361-364

ANSYS Ver 102004 Finite Element Solver for Multiphysics Problems. URL: http://www.ansys.com

Bao M H 2000 Resonant sensors and vibratory gyroscopes: Chapter 9 Handbook of Sensors and Actuators, (Amsterdam: Elsevier)

Fedder G K 1994 Simulation of microelectomechanical systems. Ph.D thesis, University of California Jeong C, Seok S, Lee B, Kim H, Chun K 2004 A study on resonant frequency and Q factor tuning for MEMS vibratory gyroscopes. J. Micromech. Microeng. 14: 1530-1536

Kwok P 1999 Fluid effects in vibrating micromachined structures, Master's thesis, Massachusetts Institute of Technology

Madou M 1997 Fundamentals of Microfabrication, (Florida: CRC Press)

Maluf N 2000 Introduction to micro electro mechanical systems engineering, (Boston: Artech house)

Patil N 2006 Design and analysis of angular rate sensors, Master's thesis, Indian Institute of Science

Polytec reference manual. URL: http://www.polytec.com

Yazdi N et al 1998 Micromachined inertial sensors, Proc. IEEE 86: 1640-1659 